\title{
CDISC CDASH Concomitant Medication Dose Units Terminology
}

National Cancer Institute

\section{Source}

National Cancer Institute. CDISC CDASH Concomitant Medication Dose Units

Terminology. NCI Thesaurus. Code C78417.

A terminology subset of the CDISC Study Data Tabulation Model (SDT M) Unit codelist created for Clinical Data Acquisition Standards Harmonization (CDASH) Concomitant Medication Dose Units terminology. 